\title{
Análisis de la rentabilidad financiero- fiscal de los planes de pensiones $y$ otros productos de captación de ahorro a largo plazo
}

\author{
Yaiza García Padrón* \\ Juan García Boza**
}

\begin{abstract}
Resumen
Entre las diversas alternativas de ahorro, previsión y/o inversión a largo plazo, un sujeto a la hora de discriminar entre ellos debe elegir aquella que le proporcione una mayor rentabilidad financiero-fiscal, para lo cual no sólo debe tomar en cuenta la rentabilidad del producto sino también su fiscalidad. Por ello, este trabajo presenta un análisis en términos homogéneos de la rentabilidad financiero-fiscal en España de los planes de pensiones, de los seguros de vida y de los fondos de inversión.
\end{abstract}

Palabras clave: rentabilidad financiero-fiscal, planes de pensiones, seguros de vida, fondos de inversión.

JEL: H24, H39, G22, G23

\section{Introducción}

T a incertidumbre existente sobre el nivel de sostenimiento de las pensiones Lúblicas impulsa cada vez a más personas a destinar parte de sus ahorros a diversos productos con el objetivo principal de asegurar que cuando llegue el

* Profesora investigadora del Departamento de Economía Financiera y Contabilidad de la Facultad de Ciencias Económicas y Empresariales de la Universidad de Las Palmas de Gran Canaria, España. Correo electrónico: ygarcia@empresariales.ulpgc.es

**Profesor investigador del Departamento de Economía Financiera y Contabilidad de la Facultad de Ciencias Económicas y Empresariales de la Universidad de Las Palmas de Gran Canaria, España. Correo electrónico: jgboza@defc.ulpgc.es 
Yaiza García Padrón

Juan García Boza

momento de su jubilación puedan disponer de un determinado capital ${ }^{1}$. Entre éstos destacan los planes de pensiones, así como otros productos de ahorro y/o inversión a largo plazo como son los planes de previsión asegurados, los planes de jubilación, los Unit Linked y los fondos de inversión; en consecuencia, el sujeto debe discriminar entre ellos en función de su rentabilidad financiero-fiscal. Por ello, este trabajo se dedica al análisis financiero-fiscal en España de los mencionados productos, cuando el sujeto partícipe o tomador coincide en la misma persona con el beneficiario.

El trabajo se ha estructurado en cinco apartados: después de la introducción, en la segunda sección se realiza un análisis financiero-fiscal de los planes de pensiones en donde se determinan los modelos matemáticos-financieros que permitan obtener la rentabilidad efectiva para el sujeto; en la tercera sección se estudian diversos contratos de seguros de vida, concretamente los planes de previsión asegurados, los planes de jubilación y los Unit Linked; asimismo, se expone el cálculo de la rentabilidad financiero-fiscal aparejada a cada uno de ellos; posteriormente, en la cuarta sección se analizan los fondos de inversión, tanto de reparto como de capitalización, y se determina la rentabilidad real que obtendrá un sujeto que invierta en ellos; por último, en la quinta sección se exponen las conclusiones más relevantes del estudio.

\section{Planes de pensiones}

En España legalmente no se ha dado una definición exacta de los planes de pensiones, sino que ésta atiende principalmente a la función que realizan. Así, en el artículo 1.1 del Texto Refundido de la Ley de Regulación de los Planes y Fondos de Pensiones, aprobado por el Real Decreto Legislativo 1/2002, de 29 de noviembre, se estipula que "[...] los planes de pensiones definen el derecho de las personas a cuyo favor se constituyen, a percibir rentas o capitales por jubilación, supervivencia, viudedad [sic], orfandad o invalidez [... $]^{\prime 2}$.

\footnotetext{
${ }^{1}$ En esta línea, Alvira et al. (1996:90) señalan que se ha producido un cambio en las creencias y valores de los españoles, quienes han perdido confianza en el sistema público y ello se traduce en intenciones de suscribir planes de pensiones.

${ }^{2}$ Un estudio respecto al carácter contractual de los planes de pensiones se puede encontrar en Sánchez et al. (1989:15-34).
} 
Análisis de la rentabilidad financiero-fiscal de los planes de pensiones

y otros productos de captación de ahorro a largo plazo

Las características fundamentales de los planes de pensiones son la voluntariedad de constitución y adhesión, su naturaleza jurídica-privada, y su carácter complementario no sustitutivo de la seguridad social; también se ha de destacar su iliquidez al no poderse rescatar las aportaciones realizadas.

Por tanto, los planes de pensiones se configuran como instituciones de previsión voluntaria, libre, privada y en ocasiones de carácter complementario, aunque nunca sustitutivo, al sistema público de previsión social en los que contractualmente los partícipes se comprometen a realizar aportaciones con el fin de constituir un capital para cuando llegue el momento de su jubilación.

Asimismo, las cuantías aportadas a los planes se deben integrar en un fondo de pensiones que realizará la inversión de éstas, configurándose los mismos como patrimonios independientes, sin personalidad jurídica, que constituyen el medio para instrumentar los planes de pensiones. Por tanto, los planes y fondos de pensiones son dos figuras estrechamente vinculadas que se complementan y se necesitan mutuamente para existir.

En este sentido, los planes de pensiones implican retrasar el consumo actual de forma prolongada en el tiempo para poder realizar un acto de consumo futuro; sin embargo, esta espera requiere que los sujetos estén bien asesorados y sean precavidos con respecto a las cuantías que aportan a los planes debido a la imposibilidad de poder disponer de las cantidades satisfechas ni siquiera ante alguna urgencia o difícil situación, salvo en los supuestos estipulados de enfermedad grave o de desempleo de larga duración. En consecuencia, la falta de liquidez en los planes de pensiones conlleva muchas veces a la reticencia de los sujetos, la cual es compensada a través de las ventajas fiscales que tiene adheridas este producto. Por todo lo anterior, a la hora de analizar un plan de pensiones como producto de ahorro, inversión y previsión frente a otras alternativas se debe estudiar su rentabilidad financiero-fiscal, dado que ésta es el tanto de interés anual de la ley de capitalización compuesta que verifica la ecuación de equivalencia financiera entre el conjunto de prestaciones desembolsadas por el inversor y el conjunto de contraprestaciones recibidas tomando en cuenta la tributación correspondiente, en la que se encuentran las deducciones y bonificaciones fiscales aplicables. 
Yaiza García Padrón

Juan García Boza

Debido a la gran variedad de situaciones que pueden contemplarse y con el fin de delimitar el marco de estudio del análisis financiero fiscal se establecen las siguientes hipótesis:

- Se analizan planes de pensiones del sistema individual.

- Las aportaciones se realizan a final de año y su cuantía está siempre dentro de los límites fiscales.

- El partícipe realiza aportaciones periódicas hasta el acaecimiento de alguna de las contingencias previstas.

- La liquidación del Impuesto sobre la Renta de las Personas Físicas se realiza a final del mes de junio.

- La contingencia ocurre a principios de año.

- No existen gastos ni comisiones.

- Si las prestaciones se perciben en forma de renta se considera con periodicidad anual.

Para este estudio es necesario tener presente las características fiscales de los planes de pensiones, de las cuales las más significativas son la deducibilidad de las aportaciones en el Impuesto sobre la Renta y la existencia de un límite máximo a la aportación anual ${ }^{3}$; y en cuanto a las prestaciones, que éstas se gravan como rendimientos del trabajo personal $1^{4}$. En los cuadros 1 y 2 se recoge de manera sintética el régimen fiscal de las aportaciones y prestaciones de los planes de pensiones en España ${ }^{5}$. Además, con respecto a esta última se ha de señalar que no existe un tipo de retención común para todos $\operatorname{los}$ sujetos $^{6}$ al contrario de lo que ocurre en otros productos financieros, por ejemplo, los planes de jubilación, los Unit Linked, los fondos de inversión o los depósitos.

3 Según Sanz (1996:62) una de los motivos de que los planes de pensiones no hayan alcanzado todavía su desarrollo esperado en España son las excesivas limitaciones interpuestas por su normativa reguladora como son su iliquidez y los límites máximos de aportaciones.

${ }^{4}$ Conforme con lo estipulado en el artículo 16.2 del Texto Refundido de la Ley del Impuesto sobre de la Renta de las Personas Físicas, aprobado por el Real Decreto Legislativo 3/2004, del 5 de marzo.

5 Recogidos en el Texto Refundido de la Ley del Impuesto sobre de la Renta de las Personas Físicas, aprobado por el Real Decreto Legislativo 3/2004, del 5 de marzo, en los artículos 50.1, 60.1, 60.5 y 61.1 para las aportaciones, y en los artículos 16.2.a, 17.2.b, 17.2.c y 17.3 para las prestaciones.

6 A la hora de determinar el importe correspondiente al ingreso a cuenta se debe seguir el procedimiento estipulado para los rendimientos del trabajo, contemplado en los artículos 75-85 del Reglamento del Impuesto sobre la Renta de las Personas Físicas, aprobado por el Real Decreto 1775/2004, del 30 de julio. 
Análisis de la rentabilidad financiero-fiscal de los planes de pensiones y otros productos de captación de ahorro a largo plazo

\section{Cuadro 1}

Régimen fiscal actual de las aportaciones a los planes de pensiones

\begin{tabular}{|c|c|c|c|}
\hline Sujeto & \multicolumn{3}{|c|}{ Tratamiento fiscal } \\
\hline \multirow{5}{*}{ Partícipe } & \multicolumn{3}{|c|}{$\begin{array}{l}\text { - Deducible en la base imponible (BI) del Impuesto de la Renta de las } \\
\text { Personas Físicas sin convertirla en negativa. } \\
\text { - Exceso de aportación deducible en BI correspondiente a los } 5 \text { ejercicios } \\
\text { siguientes. }\end{array}$} \\
\hline & \multirow{4}{*}{$\begin{array}{l}\text { - } \quad \text { Límite } \\
\text { anual }\end{array}$} & \multicolumn{2}{|r|}{$8.000 €$} \\
\hline & & $\begin{array}{l}\text { Minusválidos: } 24.250 € \\
\quad \text { (propia + familiar) }\end{array}$ & $\begin{array}{l}\text { - Aportación propia: } 24.250 € . \\
\text { - Aportación de un familiar: } 8.000 € .\end{array}$ \\
\hline & & Mayores de 52 años: & $\begin{array}{l}\text { - } 1.250 € \text { adicionales por cada año que } \\
\text { exceda de } 52 \text { años. } \\
\text { - Personas de } 65 \text { años o más: } 24.250 € \text {. }\end{array}$ \\
\hline & & \multicolumn{2}{|c|}{ Cónyuge (con rentas inferiores a $8.000 €$ ): $2.000 €$. } \\
\hline
\end{tabular}

Fuente: Elaboración propia.

\section{Cuadro 2}

Régimen fiscal actual de las prestaciones de los planes de pensiones

\begin{tabular}{|c|c|c|c|}
\hline Materialización & \multicolumn{3}{|c|}{ Tratamiento fiscal } \\
\hline \multirow[b]{2}{*}{ Renta } & \multirow[b]{2}{*}{$\begin{array}{l}\text { Rendimiento } \\
\text { del trabajo }\end{array}$} & \multicolumn{2}{|l|}{ No reducción. } \\
\hline & & \multicolumn{2}{|c|}{$\begin{array}{l}\text { - Minusválido: reducción hasta un importe máximo de } 2 \\
\text { veces el salario mínimo interprofesional. }\end{array}$} \\
\hline \multirow{2}{*}{ Capital } & \multirow{2}{*}{$\begin{array}{l}\text { Rendimiento } \\
\text { del trabajo }\end{array}$} & $\begin{array}{l}\text { - Transcurrido más de } \\
\text { dos años desde la } \\
\text { primera aportación: }\end{array}$ & $\begin{array}{l}\text { - Reducción del 40\%. } \\
\text { - Minusválido: reducción del 50\%. }\end{array}$ \\
\hline & & $\begin{array}{l}\text { - Sin transcurrir más } \\
\text { de dos años desde la } \\
\text { primera aportación: }\end{array}$ & $\begin{array}{l}\text {-No reducción. } \\
\text {-Contingencia por invalidez: } \\
\text { reducción del } 40 \% \text {. }\end{array}$ \\
\hline
\end{tabular}

Fuente: Elaboración propia. 
Yaiza García Padrón Juan García Boza

El diagrama de esta operación financiera se puede dividir en dos partes en función de si los flujos de capitales se derivan de las aportaciones o de las prestaciones. Por un lado, están las aportaciones anuales del partícipe al Plan $P_{s}$ y los ahorros fiscales derivados de la deducción en el Impuesto sobre la Renta por las aportaciones realizadas: $A_{s+\frac{1}{2}}=P_{s} \cdot t_{s}$, siendo $t_{s}$ el tipo de gravamen del partícipe, tal y como se puede ver en la figura 1.

\section{Figura 1}

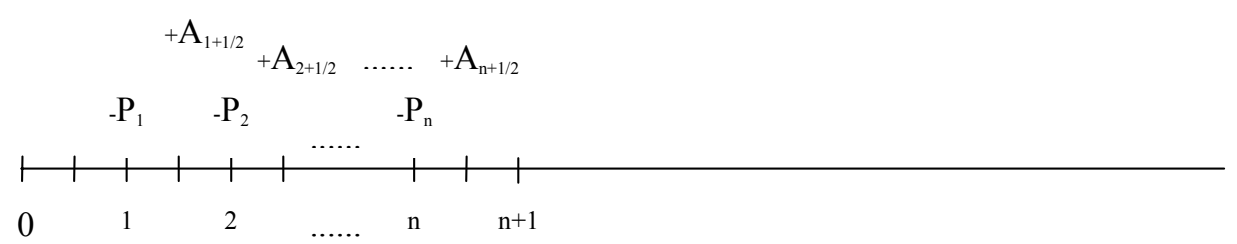

Por otra parte, están las prestaciones del plan y su tributación. En este análisis se van a estudiar diversas operaciones en función de cómo se perciban las prestaciones, si es en forma de renta, en forma de capital o en forma mixta.

Así, si las prestaciones del plan $C_{n+d+h}$ constituyen una renta anual pospagable, a final de cada año se percibirán las prestaciones netas:

$C_{n+d+h}^{*}=C_{n+d+h}-R_{n+d+h}$, una vez detraída la retención correspondiente $R_{n+d+h \text {. }}$ Además, a mitad de cada periodo el sujeto deberá satisfacer la cuota del Impuesto sobre la Renta derivada de las cuantías percibidas ${ }^{7}$ :

$T_{n+d+h+\frac{1}{2}}=C_{n+d+h} \cdot t_{n+d+h}-R_{n+d+h}$, siendo $t_{n+d+h}$ el tipo de gravamen del sujeto en el periodo $n+d+h$. Por tanto, el diagrama temporal de esta operación financiera, en el caso de que se difiera la percepción de las prestaciones ${ }^{8}$, se presenta en la figura 2:

\footnotetext{
7 Si el sujeto fuese un minusválido tendría derecho a una reducción de hasta un importe máximo de dos veces el salario mínimo interprofesional. En este caso su cuota fiscal se calcularía a través de la siguiente expresión: $T_{n+d+h+\frac{1}{2}}=\left(C_{n+d+h}-D_{n+d+h}\right) \cdot t_{n+d+h}-R_{n+d+h}$, donde $D_{n+d+h}$ es la reducción en el año $n+d+h$.
}

8 Si no existe diferimiento $d=0$. 
Análisis de la rentabilidad financiero-fiscal de los planes de pensiones y otros productos de captación de ahorro a largo plazo

Figura 2

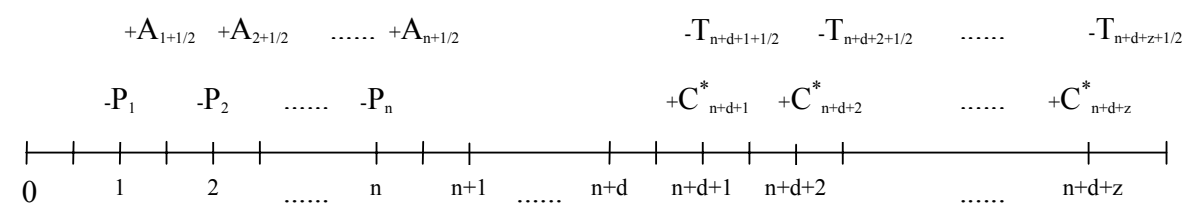

A través de la ecuación de equivalencia financiera entre todos los cobros y pagos, planteada en el punto $n$, y de acuerdo con el sistema de capitalización compuesta se obtiene la rentabilidad financiero-fiscal $\left(i_{a}\right)$, ecuación 1:

$$
\begin{aligned}
\sum_{s=1}^{n} P_{s} \cdot\left(1+i_{a}\right)^{n-s} & -\sum_{s=1}^{n} A_{s+\frac{1}{2}} \cdot\left(1+i_{a}\right)^{n-\left(s+\frac{1}{2}\right)}= \\
& =\sum_{h=1}^{z} C_{n+d+h}^{*} \cdot\left(1+i_{a}\right)^{-(d+h)}-\sum_{h=1}^{z} T_{n+d+h+\frac{1}{2}} \cdot\left(1+i_{a}\right)^{-\left(d+h+\frac{1}{2}\right)}
\end{aligned}
$$

siendo $z$ el número de años durante los cuales el beneficiario percibirá la renta.

Si en cambio, las prestaciones del plan se perciben a través de un único capital y ha transcurrido más de dos años desde que se realizó la primera aportación, se tributa por el $60 \%$ de la prestación, calculándose la cuota tributaria a través de la siguiente expresión ${ }^{9}: T_{n+d+1+\frac{1}{2}}=C_{n+d+1} \cdot 0,6 \cdot t_{n+d+1}-R_{n+d+1}$. En este caso la operación financiera quedaría definida como se expresa en el diagrama temporal siguiente, figura 3 , y su rentabilidad se determina a través de la ecuación de equivalencia financiera 2 :

\footnotetext{
Si cuando se devenga la prestación no ha transcurrido más de dos años desde que se realizó la primera aportación y aquélla se percibe en forma de capital, no se tendría derecho a reducción alguna, en consecuencia, desaparecería del cálculo de la cuota tributaria el coeficiente multiplicador 0,6 , excepto en el caso de que las prestaciones se perciban por el acaecimiento de la contingencia de invalidez.
} 
Yaiza García Padrón

Juan García Boza

\section{Figura 3}

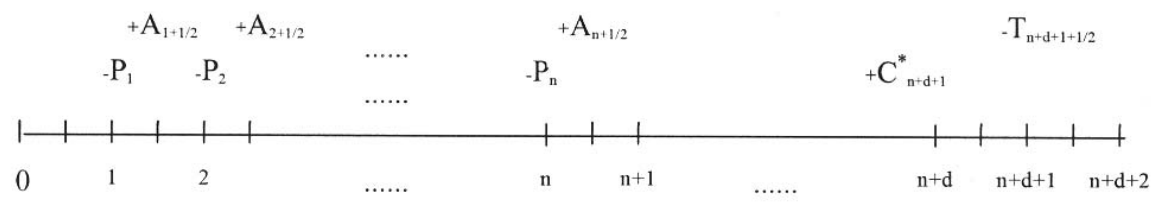

Donde se representa por $C_{n+d+1}^{*}$ al capital neto que se percibirá en $n+d+1$ una vez detraída la retención correspondiente: $C_{n+d+1}=C_{n+d+1}-R_{n+d+1}$

$$
\begin{aligned}
\sum_{s=1}^{n} P_{s} \cdot\left(1+i_{a}\right)^{n-s} & -\sum_{s=1}^{n} A_{s+\frac{1}{2}} \cdot\left(1+i_{a}\right)^{n-\left(s+\frac{1}{2}\right)}= \\
& =C_{n+d+1}^{*} \cdot\left(1+i_{a}\right)^{-d}-T_{n+d+1+\frac{1}{2}} \cdot\left(1+i_{a}\right)^{-\left(d+1+\frac{1}{2}\right)}
\end{aligned}
$$

Sin embargo, si las prestaciones del Plan se materializan en forma mixta, una parte a través de un capital y el resto mediante una renta, la operación financiera resultante será una combinación de los casos expuestos, presentándose un ejemplo de la misma en el supuesto que la parte que se perciba en forma de renta sea a través de cuantías pospagables. En la figura 4 se encuentra el diagrama de esta operación:

\section{Figura 4}

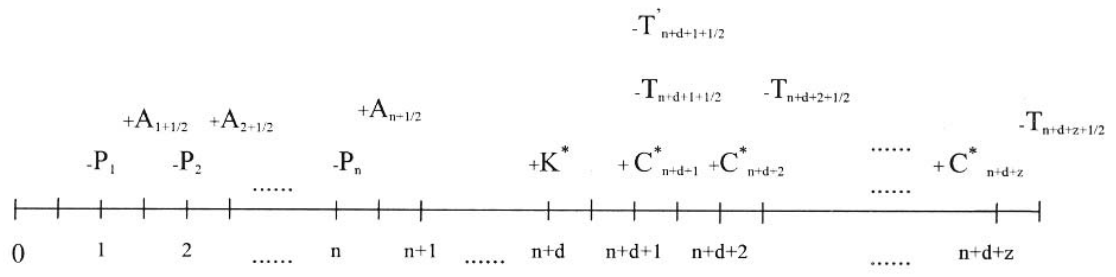


Análisis de la rentabilidad financiero-fiscal de los planes de pensiones y otros productos de captación de ahorro a largo plazo

Donde, por un lado, $K^{*}$ es la cuantía neta correspondiente a la prestación en forma de capital, percibida al principio del año $n+d+1$, una vez minorada por la retención correspondiente, y $T_{n+d+1+\frac{1}{2}}^{\prime}$ su cuota tributaria. Por otro lado, $C_{n+d+h}^{*}$ son las prestaciones netas que se perciben en forma de renta una vez detraída la retención correspondiente y $T_{n+d+h+\frac{1}{2}}$ su cuota tributaria. Tales cuotas fiscales se calculan de forma similar a la especificada en el caso de que se perciban las prestaciones del Plan a través de un único capital o en forma de renta, respectivamente. La expresión analítica que recoge esta operación financiera se muestra en la ecuación 3:

$$
\begin{aligned}
& \sum_{s=1}^{n} P_{s} \cdot\left(1+i_{a}\right)^{n-s}-\sum_{s=1}^{n} A_{s+\frac{1}{2}} \cdot\left(1+i_{a}\right)^{n-\left(s+\frac{1}{2}\right)}= \\
& =K^{n} \cdot\left(1+i_{a}\right)^{-d}-T_{n+d+1+\frac{1}{2}}^{\prime} \cdot\left(1+i_{a}\right)^{-\left(d+1+\frac{1}{2}\right)}+\sum_{h=1}^{z} C_{n+d+h}^{*} \cdot\left(1+i_{a}\right)^{-(d+h)}-\sum_{h=1}^{z} T_{n+d+h+\frac{1}{2}} \cdot\left(1+i_{a}\right)^{-\left(d+h+\frac{1}{2}\right)}
\end{aligned}
$$

Un caso particular por destacar en este estudio son los planes de pensiones garantizados cuando se ejerce la garantía, éstos son planes que través de la contratación de seguros ofrecen una revalorización asegurada a un plazo determinado. En este sentido, si llegada la fecha prefijada en el compromiso de revalorización y el valor de los derechos consolidados del plan es inferior a la cuantía garantizada, la entidad aseguradora que cubre la garantía tendrá que abonar al sujeto un importe por la diferencia, considerándose éste un rendimiento del capital mobiliario ${ }^{10}$. En esta línea, a continuación en la figura 5 se muestra esta operación financiera y en la ecuación 4 se presenta la determinación de la rentabilidad financiero-fiscal $\left(i_{a}\right)$ de los planes de pensiones garantizados bajo este supuesto y en el caso que coincida la fecha de revalorización con el devengo de las prestaciones del plan y que las prestaciones se perciban en forma de capital sin diferimiento.

\footnotetext{
10 Si no se ejerce la garantía, es decir, si todas las cantidades percibidas por el beneficiario proceden del propio plan de pensiones, la fiscalidad de estos planes no difiere del resto de planes de pensiones del sistema individual, siendo idéntico el estudio de su rentabilidad financiero-fiscal.
} 
Yaiza García Padrón

Juan García Boza

\section{Figura 5}

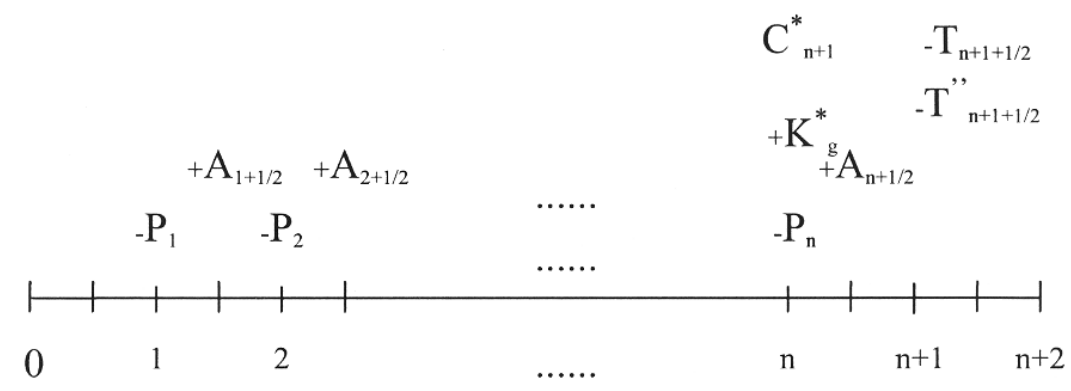

$$
\begin{aligned}
\sum_{s=1}^{n} P_{s} \cdot\left(1+i_{a}\right)^{n-s}- & \sum_{s=1}^{n} A_{s+\frac{1}{2}} \cdot\left(1+i_{a}\right)^{n-\left(s+\frac{1}{2}\right)}= \\
& =C_{n+1}^{*}-T_{n+1+\frac{1}{2}} \cdot\left(1+i_{a}\right)^{-\left(1+\frac{1}{2}\right)}+K_{g}^{*}-T_{n+1+\frac{1}{2}}^{n} \cdot\left(1+i_{a}\right)^{-\left(1+\frac{1}{2}\right)}
\end{aligned}
$$

Denominamos $K_{g}^{*}$ al importe neto de la cuantía diferencia derivada de la garantía que percibe el sujeto al principio del año $n+1$, esto es $K_{g}^{*}=K_{g}-R_{n+1}^{\prime \prime}$, donde $K g$ es el importe íntegro del mismo obtenido como diferencia entre la cuantía garantizada y el importe de los derechos consolidados del Plan en dicha fecha y $R_{n+1}^{\prime \prime}$ la retención correspondiente: $R_{n+1}^{\prime \prime}=K_{g} \cdot 0,15$ y es la cuota tributaria derivada del importe percibido por la garantía en el año $n+1:_{n+1+\frac{1}{2}}^{T^{\prime \prime}}=K_{g} \cdot t_{n+1}-R_{n+1}^{\prime \prime}$. Por otro lado, $C_{n+1}^{*}$ es el importe de la prestación en forma de capital que percibe el beneficiario del plan al principio del año $n+1$, una vez detraída su retención y ${ }_{n+1+\frac{1}{2}}^{T}$ la cuota tributaria correspondiente a la prestación percibida en forma de capital ${ }^{11}$.

11 Esta cuota fiscal se calcula de manera similar a la ya señalada en el caso de que se perciba la prestación del plan de pensiones a través de un único capital. 
Análisis de la rentabilidad financiero-fiscal de los planes de pensiones

y otros productos de captación de ahorro a largo plazo

Por tanto, se ha de reseñar que la rentabilidad financiero-fiscal de un sujeto que suscriba un plan de pensiones diferirá en función del tipo de plan, garantizado o no, y de la forma de percepción de las prestaciones del plan, así como de las características particulares del sujeto, su edad, al variar el límite de deducción de las cuantías aportadas para sujetos de más de 52 años, y su renta, dado que ésta determinará el tipo de gravamen a aplicar en función de la escala en la que se sitúe el sujeto. En esta línea, Domínguez y López (2004:25) señalan que los factores que influyen en que un sujeto participe en un plan de pensiones del sistema individual, así como en la aportación que realice son: estado civil, ocupación, participación en un plan del sistema de empleo y su tipo marginal.

\section{Seguros de vida}

Cuando un sujeto quiere ser previsor y destinar parte de sus ahorros a un producto con el fin de constituir un determinado capital para cuando se jubile puede suscribir un plan de pensiones o un seguro de vida. Así, por la competencia que supone para los planes de pensiones del sistema individual de este producto, dentro de la gran diversidad de contratos de seguros de vida que existen, se analizan los planes de previsión asegurados, los planes de jubilación y, por último, los Unit Linked, cada uno de ellos constituye una opción de ahorro.

\section{A. Planes de previsión asegurados}

Los planes de previsión asegurados, producto muy reciente y novedoso en España, creados a través de las diversas modificaciones introducidas por la Ley 46 de 2002, del 18 de diciembre, en el Impuesto sobre la Renta de las Personas Físicas. Se definen como contratos de seguros de vida que ofrecen una rentabilidad garantizada y asegurada, que cubren las mismas contingencias que los planes de pensiones y que pueden disfrutar de la misma fiscalidad que éstos, siempre y cuando cumplan los siguientes requisitos ${ }^{12}$ : coincidan en la misma persona la figura del tomador, del asegurado y del beneficiario; sólo se permita la disposición anticipa-

12 Recogidos en el artículo 60.3 del texto refundido de la Ley del Impuesto sobre la Renta de las Personas Físicas, aprobado por el Real Decreto Legislativo 3/2004, del 5 de marzo. 
Yaiza García Padrón

Juan García Boza

da, total o parcial en los mismos casos que los establecidos para los planes de pensiones; ofrezcan obligatoriamente una garantía de interés y se utilicen técnicas actuariales. Por tanto, el planteamiento del cálculo de la rentabilidad financierofiscal de los mismos es idéntico al de los planes de pensiones ya analizado.

\section{B. Planes de jubilación}

Los planes de jubilación constituyen uno de los productos de ahorro a largo plazo que más competencia representan para los planes de pensiones del sistema individual. Son contratos de seguro de vida mixto en los que a través del pago de una prima dan derecho a cobrar una prestación al término del mismo.

Entre sus características más relevantes destacan que es el asegurado quien fija libremente la edad a la que desea percibir las prestaciones, se permite el rescate de las aportaciones, puede garantizar un interés mínimo anual y dicha rentabilidad se completa a través de la participación en los beneficios de la compañía, por sus prestaciones se tributará por rendimientos de capital mobiliario ${ }^{13}$, estando sujetas a un tipo de retención del 15\%, el cual se aplicará sobre el importe por el que se integrará en la base imponible ${ }^{14}$. Además, el modo de cuantificar dicho rendimiento y su tributación difiere en función de cómo se perciba (en forma de renta, capital o mixta).

En el análisis de la rentabilidad financiero-fiscal de los planes de jubilación se parte de las mismas hipótesis planteadas anteriormente para los planes de pensiones. Asimismo, se presenta dicho análisis para cada una de las distintas formas en que se pueden percibir las prestaciones.

Si la prestación se percibe en forma de renta para la determinación de la cuantía por computar en cada periodo como rendimiento del capital mobiliario $\left(M_{n+d+h}\right)$ se aplica a cada anualidad o prestación devengada $\left(C_{n+d+h}\right)$ una serie de porcentajes $\left(\beta_{i}\right)$, esto es: $M_{n+d+h}=\beta_{i} \cdot C_{n+d+h}$. Tales porcentajes $\beta_{i}$ son constantes en la

13 Estos rendimientos están sujetos al Impuesto sobre la Renta de las Personas Físicas siempre que contratante y beneficiario coincidan en una misma persona.

14 Su tratamiento fiscal está comprendido fundamentalmente en los artículos 23-24, 94 y 103.4 del Texto Refundido de la Ley del Impuesto sobre de la Renta de las Personas Físicas, aprobado por el Real Decreto Legislativo 3/2004, de 5 de marzo, y en su Reglamento aprobado por el Real Decreto 1775/2004, del 30 de julio, en los artículos 15-17, 19, 88 y 91 . 
Análisis de la rentabilidad financiero-fiscal de los planes de pensiones

y otros productos de captación de ahorro a largo plazo

operación, pero difieren en función de la edad del perceptor en el momento origen de la renta si se trata de una renta vitalicia o de la duración de la renta en años si se trata de una renta temporal. Estos porcentajes $\beta_{i}$ se muestran en el cuadro 3.

\section{Cuadro 3}

Porcentajes $\beta_{i}$ a aplicar en función del tipo de renta

\begin{tabular}{|l|l|}
\hline \multicolumn{1}{|c|}{ Renta vitalicia } & \multicolumn{1}{c|}{ Renta temporal } \\
\hline $45 \%$ si edad perceptor $<40$ & $15 \%$ si duración renta $\leq 5$ \\
$40 \%$ si $40 \leq$ edad perceptor $\leq 49$ & $25 \%$ si $5<$ duración $\leq 10$ \\
$35 \%$ si $50 \leq$ edad perceptor $\leq 59$ & $35 \%$ si $10<$ duración $\leq 15$ \\
$25 \%$ si $60 \leq$ edad perceptor $\leq 69$ & $42 \%$ si duración renta $>15$ \\
$20 \%$ si edad perceptor $>69$ & \\
\hline
\end{tabular}

Además, el sujeto no percibe la cuantía total de la prestación devengada $\mathrm{C}_{\mathrm{n}+\mathrm{d}+\mathrm{h}}$, sino su cuantía neta, una vez detraída la retención calculada sobre el rendimiento del capital mobiliario: $R_{n+d+h}=0,15 \cdot M_{n+d+h}$. Asimismo, a mitad de cada periodo el sujeto deberá satisfacer la cuota del Impuesto sobre la Renta derivada de las cuantías percibidas en el periodo anterior, hallándose dicha cuota de la siguiente forma: $T_{n+d+h+\frac{1}{2}}=M_{n+d+h} \cdot t_{n+d+h}-R_{n+d+h}$, siendo $t_{n+d+h}$ el tipo de gravamen del sujeto en el periodo $n+d+h$.

Por otro lado, si se trata de una renta diferida para el cálculo del rendimiento del capital mobiliario a la cuantía señalada se añadirá el rendimiento obtenido (R) hasta la constitución de la misma, calculándose en este caso la retención sobre esta cuantía, dicho rendimiento $(R)$ se obtiene a través de la siguiente expresión: 
Yaiza García Padrón Juan García Boza

$R=\frac{V_{a}-\sum_{s=1}^{n} P_{s}}{z}$, siendo $V_{a}$ el valor actual financiero-actuarial de la renta que se constituye, $\sum_{s=1}^{n} P_{s}$ el importe de las primas satisfechas y z la duración de la renta expresada en años, tomando el valor de 10 como máximo o en el caso de una renta vitalicia ${ }^{15}$.

Por tanto, los planes de jubilación presentan un tratamiento fiscal en el Impuesto sobre la Renta de las Personas Físicas muy complejo, el cual exige definir con mayor rigor diversos factores para realizar un estudio exhaustivo de su rentabilidad financiero-fiscal. Así, se ha de explicitar tanto las primas a abonar, como la modalidad de percepción de las prestaciones, además de la edad del beneficiario en el momento origen de la renta si se trata de una renta vitalicia, o la duración de la renta si es una renta temporal. A continuación, se presenta la operación financiera resultante, figura 6 , y la determinación de su rentabilidad $\left(i_{a}\right)$, ecuación 5 , si las prestaciones se perciben en forma de renta pospagable.

Figura 6

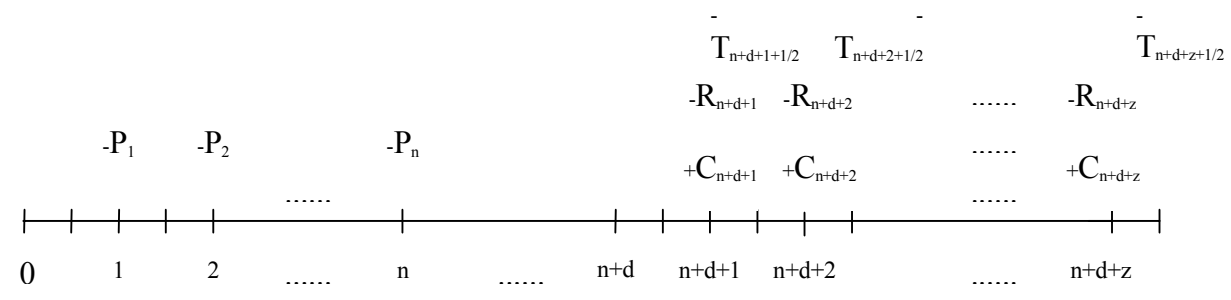

Donde se denomina $P_{s}$ a las primas anuales que se satisfacen, $C_{n+d+h}$ al importe bruto en unidades monetarias de la prestación devengada en el año $n+d+h, R_{n+d+h}$ a la cuantía de la retención derivada de la prestación percibida en el año $n+d+h$,

15 Un caso particular es el supuesto de que las prestaciones se perciban por jubilación o invalidez en forma de renta diferida y además que no haya existido ningún tipo de movilización de las provisiones del contrato durante su vigencia es cuando se permite diferir su tributación. En este caso, se integrarán en la base imponible del impuesto como rendimientos del capital mobiliario a partir del momento en que su cuantía exceda de las primas satisfechas o del valor actuarial de las rentas en el supuesto de donación; en este supuesto no se aplicarán porcentajes de reducción si las contingencias cubiertas son las mismas que las previstas en los planes de pensiones, exigiéndose además una antigüedad mínima de dos años de contrato si las prestaciones se perciben por jubilación. 
Análisis de la rentabilidad financiero-fiscal de los planes de pensiones y otros productos de captación de ahorro a largo plazo $T_{n+d+h+\frac{1}{2}}$ a la cuota tributaria derivada de la mencionada prestación, $h$ al número de años transcurridos desde el origen de la renta constituida por las prestaciones, $z$ al número de años durante los cuales el beneficiario percibe las prestaciones correspondientes y $d$ al diferimiento expresado en años. Si es una renta inmediata: $d=0$, coincidiendo el punto $n$ con el punto $n+d$.

Su rentabilidad financiero-fiscal $i_{a}$ se determina a través de la ecuación de equivalencia que relaciona el valor financiero de las primas satisfechas con el de las prestaciones recibidas y su tributación, ecuación 5:

$$
\sum_{s=1}^{n} P_{s} \cdot\left(1+i_{a}\right)^{n-s}=\sum_{h=1}^{z}\left(C_{n+d+h}-R_{n+d+h}\right) \cdot\left(1+i_{a}\right)^{-(d+h)}-\sum_{h=1}^{z} T_{n+d+h+\frac{1}{2}} \cdot\left(1+i_{a}\right)^{-\left(d+h+\frac{1}{2}\right)}
$$

En cambio, si la prestación se percibe en forma de capital, al principio del año $n+d+1$ el sujeto recibirá la prestación total minorada en el ingreso a cuenta, devengándose su cuota tributaria. Tanto la retención como la cuota tributaria se calculan sobre el rendimiento generado por las primas $M_{n+d+l}$, que en este caso al tratarse de primas periódicas se calcula mediante la ponderación del rendimiento total $^{16}(R T)$ por el coeficiente $\mathrm{a}_{s} \mathrm{y}$ por un porcentaje $\mathrm{d}_{\mathrm{i}}$ en función de su periodo de generación ${ }^{17}$. Esto es: $M_{n+d+1}=\sum_{s=1}^{n} R T \cdot \alpha_{s} \cdot \delta_{i} \cdot$ El valor de $a_{s}$ se obtiene a través de la

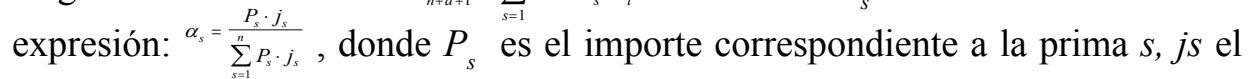
número de años transcurridos desde el pago de la prima $s$ hasta el cobro de la percepción y n el número de primas satisfechas. El valor de $d_{i}$ se muestra en el cuadro $4^{18}$ :

16 Diferencia entre el capital percibido y el importe de las primas satisfechas.

17 Existe un régimen transitorio a aplicar a las primas abonadas antes del 31 de diciembre de 1994, siempre que su tratamiento fiscal fuese el correspondiente a las variaciones patrimoniales con anterioridad a la entrada en vigor de la Ley 40/1998, de 9 de diciembre. En este caso a la prestación correspondiente a las primas se le aplicará un coeficiente reductor del $14,28 \%$ por cada año que exceda de dos, transcurridos entre la fecha de aportación de la prima y el 31 de diciembre de 1996.

18 Si la prestación se percibe por causa de invalidez, se aplicará la reducción del $75 \%$, anteriormente mencionada; si el grado de minusvalía es igual o superior al $65 \%$, en caso contrario, una reducción del $40 \%$. 
Yaiza García Padrón

Juan García Boza

\section{Cuadro 4}

Valor de $d_{i}$

$40 \%$ si primas tienen $>2$
años
$75 \%$ si primas tienen $>5$
años
$75 \%$ si prima 1 tiene $>8$
años y periodo medio
permanencia de primas $>$
4 años

En la figura 7 se explicita el diagrama temporal de la operación:

\section{Figura 7}

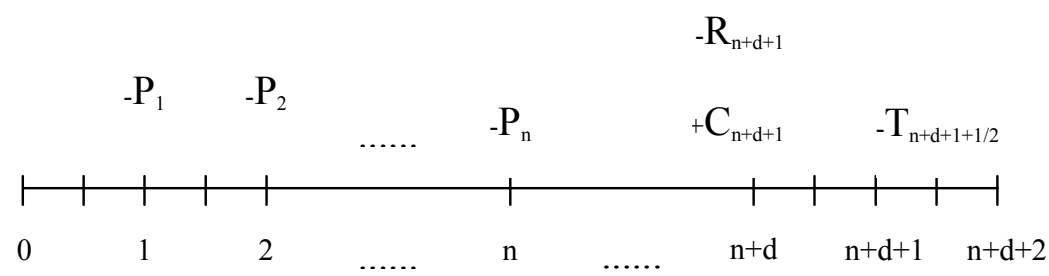

Resultando la ecuación 6 de cálculo de la rentabilidad efectiva $i_{a}$ :

$$
\sum_{s=1}^{n} P_{s} \cdot\left(1+i_{a}\right)^{n-s}=\left(C_{n+d+1}-R_{n+d+1}\right) \cdot\left(1+i_{a}\right)^{-d}-T_{n+d+1+\frac{1}{2}} \cdot\left(1+i_{a}\right)^{-\left(d+1+\frac{1}{2}\right)}
$$

En cambio, si la prestación se percibe en forma mixta, es decir, las prestaciones se materializan de forma combinada a través de rentas de cualquier modalidad con un único cobro en forma de capital, las reducciones referidas a la prestación en forma de capital sólo se pueden aplicar al cobro efectuado en dicha forma. En este 
Análisis de la rentabilidad financiero-fiscal de los planes de pensiones y otros productos de captación de ahorro a largo plazo

trabajo se analiza el supuesto de que se combine con una renta temporal anual pospagable, figura 8 , obteniéndose el modelo matemático-financiero expresivo de esta operación, ecuación 7.

\section{Figura 8}

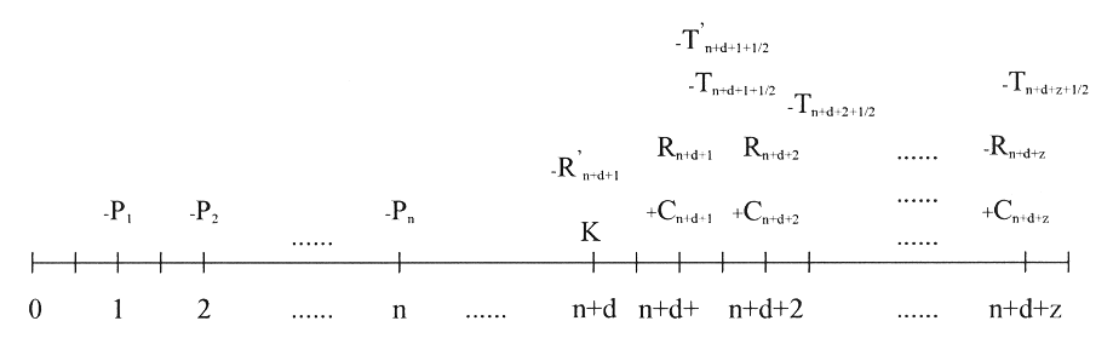

$$
\begin{aligned}
\sum_{s=1}^{n} P_{s} \cdot(1+ & \left.i_{a}\right)^{n-s}=\left(K-R_{n+d+1}^{\prime}\right) \cdot\left(1+i_{a}\right)^{-d}-T_{n+d+1+\frac{1}{2}}^{\prime} \cdot\left(1+i_{a}\right)^{-\left(d+1+\frac{1}{2}\right)}+ \\
& +\sum_{h=1}^{z}\left(C_{n+d+h}-R_{n+d+h}\right) \cdot\left(1+i_{a}\right)^{-(d+h)}-\sum_{h=1}^{z} T_{n+d+h+\frac{1}{2}} \cdot\left(1+i_{a}\right)^{-\left(d+h+\frac{1}{2}\right)}
\end{aligned}
$$

Donde $K$ es la cuantía íntegra de la prestación que se percibe en forma de capital al principio del año $n+d+1, R_{n+d+1}^{\prime}$ el importe de la retención correspondiente a la prestación que se percibe en forma de capital y ${ }_{n+d+1+\frac{1}{2}}^{T}$ la cuota tributaria correspondiente a la prestación percibida en forma de capital al principio del año $n+d+1$.

Por tanto, si se comparan los planes de jubilación con los planes de pensiones tenemos que, a pesar de que en los planes de jubilación no se puedan deducir las primas satisfechas y sí en los planes de pensiones, los planes de jubilación presen- 
Yaiza García Padrón

Juan García Boza

tan como ventajas frente a los de pensiones que, por un lado, poseen mayor liquidez al permitir a los suscriptores retirar el dinero aportado y, por otro, que pueden garantizar un interés mínimo anual.

\section{Unit Linked}

Los Unit Linked son un seguro de vida en el que los fondos en que se materializan las provisiones técnicas se invierten en nombre y por cuenta del asegurador en un conjunto de activos, generalmente, en instituciones de inversión colectiva, elegidos por el tomador entre los estipulados en el contrato de seguro, en los cuales se ha de destacar que es el tomador quien asume el riesgo de la inversión, no existiendo por tanto diferimiento en la percepción y siendo ésta en forma de capital. Además, se permite la reestructuración de la cartera sin repercusión fiscal, así como el rescate de las primas abonadas.

Los Unit Linked pueden someterse a la misma tributación que los planes de jubilación, en donde el tomador no asume el riesgo de inversión y puede disfrutar de sus ventajas fiscales siempre y cuando cumplan determinados requisitos ${ }^{19}$, coincidiendo en ese caso el planteamiento de su rentabilidad con el de los planes de jubilación; en cambio, si no se cumplen esos requisitos ésta diferirá al supuesto objeto de análisis.

En este caso, en relación con su fiscalidad ${ }^{20}$, el tomador debe imputar anualmente, en la base imponible del Impuesto sobre la Renta de las Personas Físicas ${ }^{21}$, como rendimiento del capital mobiliario la cuantía $M_{s}$, calculado como:

19 Requisitos establecidos en el artículo 14.2.h del Texto Refundido de la Ley del Impuesto sobre de la Renta de las Personas Físicas, aprobado por el Real Decreto Legislativo 3/2004, de 5 de marzo. Si se tiene en cuenta lo ya señalado, tales requisitos se pueden resumir en que las provisiones matemáticas se inviertan en: acciones o participaciones de instituciones de inversión colectiva, conjuntos de activos reflejados de forma separada en balance de entidad aseguradora siempre que la entidad aseguradora determine activos integrantes y no se invierta en bienes inmuebles y derechos reales inmobiliarios.

20 Explicitada en los artículos 14.2.h, 23.3 y 94.3 del Texto Refundido de la Ley del Impuesto sobre de la Renta de las Personas Físicas, aprobado por el Real Decreto Legislativo 3/2004, del 5 de marzo.

21 A objeto de comparación con los Planes de Pensiones del sistema individual, así como para analizar la rentabilidad financiero-fiscal se parte del supuesto de que el tomador es una persona física. 
Análisis de la rentabilidad financiero-fiscal de los planes de pensiones y otros productos de captación de ahorro a largo plazo

$M_{s}=V_{L_{s}}-V_{L_{s-1}}$, siendo $V_{L_{s}}$ y $V_{L_{s-1}}$ el valor liquidativo total de los activos afectos a la póliza al final del periodo impositivo $s$ y $s$-1, respectivamente. Por tanto, la cuota tributaria derivada de dicho rendimiento sería $T_{s+\frac{1}{2}}=M_{s} \cdot t_{s}$ siendo $t_{s}$ el tipo de gravamen del sujeto en el periodo $s$.

Sin embargo, cuando se perciban las prestaciones del contrato para determinar el importe por computar como rendimiento del capital mobiliario, en el Impuesto sobre la Renta de las Personas Físicas ${ }^{22}$, el rendimiento derivado de dicha percepción $C_{g}$ se minorará por las cuantías, derivadas de este contrato, que se hubiesen imputado anualmente como rendimiento del capital mobiliario. En este caso, no se pueden aplicar las reducciones expuestas para los planes de jubilación. Además, la percepción de tales cuantías también está sujeta a un tipo de retención del 15\%. Por tanto, la cuota tributaría sería la siguiente: $T_{n+1+\frac{1}{2}}=\left(C_{g}-\sum_{s=1}^{n} M_{s}\right) \cdot t_{n+1}-R_{n+1}$, calculándose el rendimiento percibido $C_{g}$ como: $C_{g}=C_{n+1}-\sum_{s=1}^{n} P_{s}$, donde $C_{n+1}$ es la cuantía íntegra total de la prestación de la operación y $P_{s}$ la prima abonada en el año $s$, y la retención $R_{n+1}$ o ingreso a cuenta se obtiene como: $R_{n+1}=\left(C_{g}-\sum_{s=1}^{n} M_{s}\right) \cdot 0,15$.

De acuerdo con lo expuesto, a continuación se plantea el cálculo de la rentabilidad financiero-fiscal $\left(i_{a}\right)$ derivada de la inversión en este producto y a fin de realizar un análisis homogéneo para todos los productos se parte de las mismas hipótesis que las planteadas en la sección 2 para los planes de pensiones del sistema individual.

El diagrama de esta operación financiera, figura 9, sería el siguiente: por un lado, se encuentran las cuantías invertidas, denominando $P_{s}$ a la cuantía invertida o prima abonada en el año $s$, y la tributación $T_{S+\frac{1}{2}}$ como rendimiento del capital mobiliario por la variación anual del valor liquidativo total de los activos afectos a la póliza, ya explicitado su cálculo anteriormente. Por otro lado, en $n+1$ se percibirá la prestación del contrato $C_{n}+1$ detrayendo la retención correspondiente $R_{n}+1$, y se devenga su cuota fiscal $T_{n+1+\frac{1}{2}}$, la cual se calcula sobre la diferencia entre el rendimiento percibido y las cuantías por las que se ha tributado a lo largo

22 Siempre y cuando el tomador y el beneficiario sean la misma persona física, si no se estará sujeto al Impuesto sobre Sucesiones y Donaciones. 
Yaiza García Padrón

Juan García Boza

de la operación. Planteando la ecuación 8 de equivalencia financiera de los flujos de capitales se obtiene la rentabilidad financiero-fiscal ia de un sujeto económico que invierta en los Unit Linked:

\section{Figura 9}

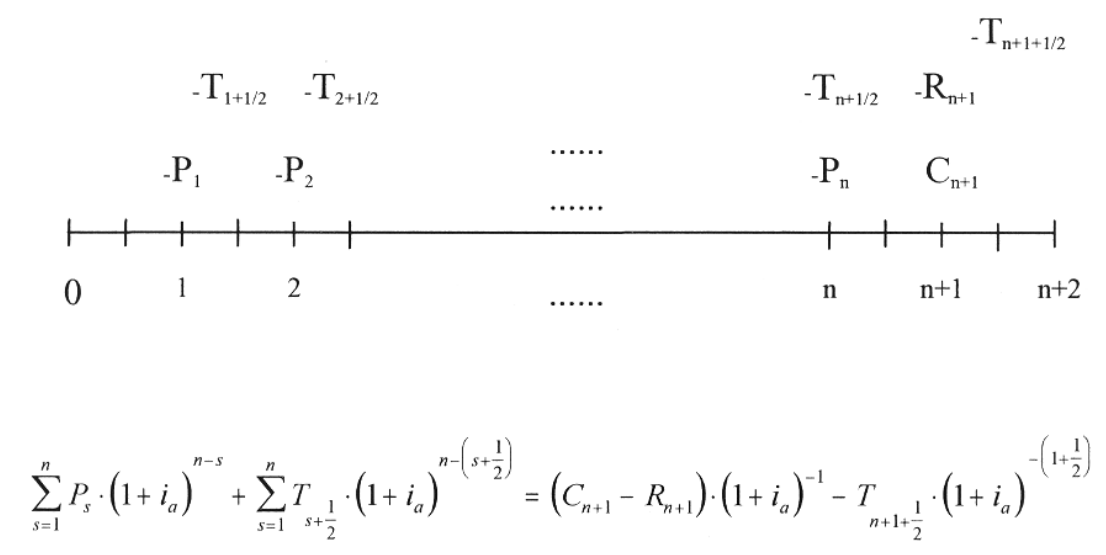

donde $n$ es el número de años que dura el contrato.

A modo de conclusión de este apartado se puede afirmar que los Unit Linked son un producto que permite combinar dos operaciones en una, al implicar la suscripción de un seguro de vida junto con la inversión en una cartera de activos, constituida principalmente por fondos de inversión y/o activos financieros, lo que conlleva mayores comisiones. La diferencia fundamental con respecto a otros productos de ahorro expuestos es que, en este caso, el riesgo financiero lo asume el tomador y no la entidad aseguradora. Además, tampoco existe una rentabilidad garantizada, a diferencia de los planes de previsión asegurados y los planes de jubilación. No obstante, los Unit Linked junto con los planes de jubilación, frente a los planes de pensiones del sistema individual, constituyen uno de los productos al que se pueden dirigir los sujetos con el objeto de planificar su ahorro a largo plazo, sin renunciar a la posibilidad de disponer de las cuantías aportadas. 
Análisis de la rentabilidad financiero-fiscal de los planes de pensiones

y otros productos de captación de ahorro a largo plazo

\section{Fondos de inversión}

Los fondos de inversión son una modalidad de institución de inversión colectiva que se configura como patrimonios pertenecientes a una pluralidad de inversores cuyo objeto exclusivo es la inversión y gestión de forma conjunta de activos financieros, cuyas características más relevantes son que existe libertad de salida a través de la venta de las participaciones, que la movilización de recursos está exenta de tributación y que las cuantías que se invierten no se pueden deducir.

Con el fin de realizar un estudio en términos homogéneos, en su análisis financiero fiscal se parte de hipótesis similares a las planteadas en la sección 2, así como se considera que todas las participaciones se venden al final del año $n+1$, distinguiendo dos casos en función de la distribución de los rendimientos: 1) fondos de reparto si los beneficios obtenidos se distribuyen periódicamente en forma de dividendos y 2) fondos de capitalización si los beneficios se acumulan al capital incrementando el valor de la participación.

\section{A. Fondos de inversión de reparto}

En los fondos de inversión de reparto los sujetos deben tributar tanto a lo largo de la operación como cuando finalice la misma por rendimientos del capital mobiliario ${ }^{23}$.

Así, con respecto a su operación financiera se encuentran, por un lado, antes de la venta de las participaciones, la cuantía $P_{s}$ que se invierte en el fondo en el año $s$ y el dividendo bruto anual que reparte el fondo $D i v_{s}$ y la retención a cuenta del dividendo percibido $R_{s}$, obtenida como: $R_{s}=\operatorname{Div} \cdot 0,15$. Además, a mitad de cada periodo se ha de tributar por los dividendos percibidos en el periodo anterior, siendo la cuota fiscal devengada: ${ }_{s+\frac{1}{2}}=\operatorname{Div}_{s} \cdot\left(p-d^{\prime}\right) \cdot t_{s}-R_{s}$, donde $p$ es la ponderación en tanto por uno del dividendo bruto ${ }^{24}, d^{\prime}$ es la deducción por dividendo de la cuota expresada en tanto por uno ${ }^{25}$ y $t_{s}$ es el tipo de gravamen que le corresponde al sujeto en el periodo $s$.

\footnotetext{
23 El tratamiento fiscal de este producto se realiza conforme a lo estipulado en los artículos 23.1, 80.a, 81 y 103.4 del Texto Refundido de la Ley del Impuesto sobre de la Renta de las Personas Físicas, aprobado por el Real Decreto Legislativo 3/2004, del 5 de marzo.

${ }^{24} p$ toma el valor de 1 si el fondo de inversión tributa en el régimen especial de las instituciones de inversión colectiva, o el valor de 1,4 si tributa en el régimen general.

${ }_{25} d$ toma el valor de $0 \mathrm{si}$ el fondo está sometido al régimen especial, o 0,4 si es al régimen general.
} 
Yaiza García Padrón Juan García Boza

Por otro lado, cuando se vendan las participaciones, en $n+1$, se percibirá el valor de reembolso total $V_{R}$ disminuido en el importe de la retención correspondiente ${ }^{26}$ : $R_{n+1}=\left(V_{R}-V_{A}\right) \cdot 0,15$ donde $V_{A}$ es el valor total de adquisición ${ }^{27}$, y a mitad del periodo siguiente se tributará por el rendimiento generado ${ }^{28}$, calculándose la cuota tributaria a través de la siguiente expresión: ${ }_{n+1+\frac{1}{2}}^{T}=\left(V_{R}-V_{A}\right) \cdot t_{n+1}-R_{n+1}$, siendo $t_{n+1}$ el tipo de gravamen del sujeto en el periodo $n+1$. Planteando en el punto $n$ la ecuación 9 de equivalencia para la determinación de la rentabilidad financiero-fiscal del sujeto.

El diagrama de esta inversión, así como su rentabilidad financiero-fiscal $i_{a}$, se definen a continuación en la figura 10 y en la ecuación 9 , respectivamente, bajo el supuesto de que el Fondo reparta los dividendos a final de año:

Figura 10

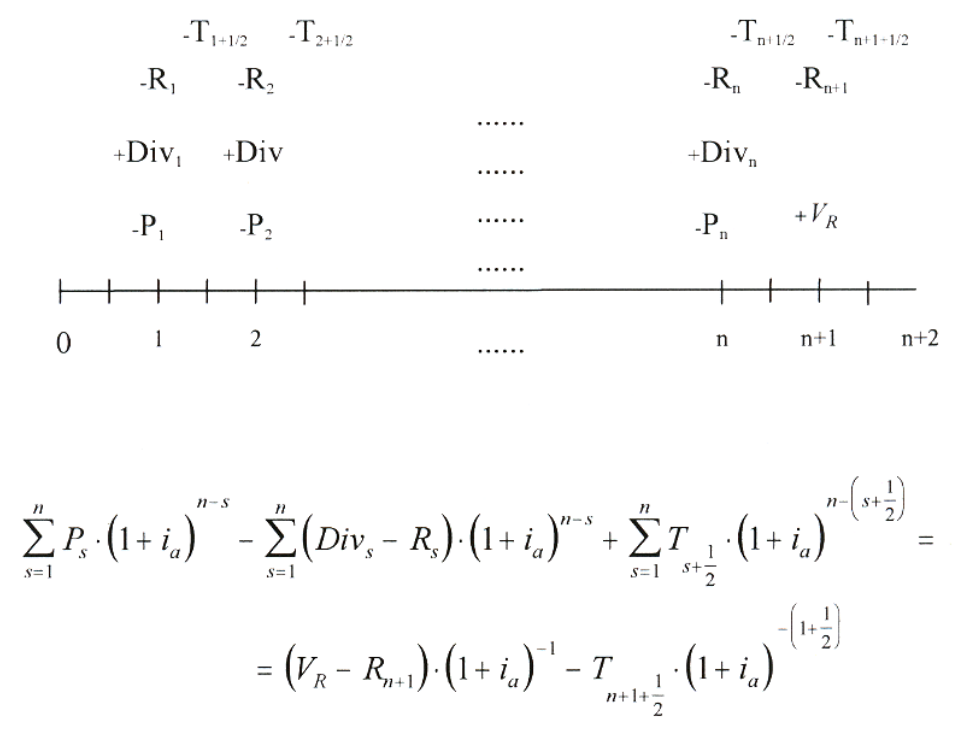

26 Siempre y cuando la diferencia expuesta sea positiva.

27 En el caso de que se posean varias participaciones de un mismo fondo de inversión y se decida vender sólo una parte de las mismas, para determinar qué valor de adquisición se debe tomar, primero se transmitirán las participaciones que se suscribieron en primer lugar, es decir, se sigue el criterio FIFO (first in first out).

28 Salvo en el caso de que los recursos se reinviertan en participaciones de otros fondos. 
Análisis de la rentabilidad financiero-fiscal de los planes de pensiones

y otros productos de captación de ahorro a largo plazo

\section{B. Fondos de inversión de capitalización}

En los fondos de capitalización, a diferencia de los fondos de reparto, sólo se tributa por la venta de las participaciones, en concepto de variación patrimonial por la diferencia entre los valores de reembolso y adquisición: $V P=V_{R}-V_{A}$, siendo similar al cálculo del rendimiento final expresado en los Fondos de Reparto y, al igual que en el caso anterior, se sigue el criterio FIFO (first in first out) ${ }^{29}$.

En este caso, la operación financiera queda definida de la siguiente forma: desde 1 hasta $n$ están las inversiones $P_{s}$ que se realicen en el fondo, y al final del año $n+1$ el sujeto percibirá el valor de la venta de las participaciones $V_{R}$ menos la retención correspondiente ${ }^{30}: R_{n+1}=\left(V_{R}-V_{A}\right) \cdot 0,15 \mathrm{y}$ finalmente se devenga la cuota tributaria cuyo cálculo varía en función del periodo de generación del rendimiento de la inversión:

$$
T_{n+1+\frac{1}{2}}=\left\{\begin{array}{l}
\left(V_{R}-V_{A}\right) \cdot t_{n+1}-R_{n+1} \text { si } g \leq 1 \\
\left(V_{R}-V_{A}\right) \cdot 0,15-R_{n+1} \text { si } g>1
\end{array}\right.
$$

siendo $g$ el número de años transcurridos entre la adquisición y la venta de las participaciones. Si dicha diferencia arrojase un signo negativo y $g \leq 1$, ésta se compensaría con el resto de ganancias patrimoniales de la parte general y, luego, hasta el $10 \%$ del saldo de los rendimientos e imputaciones de renta; la cuantía restante se compensará en los cuatro ejercicios siguientes. En cambio, si la pérdida se genera en un periodo superior al año, es decir, si g>1, ésta se compensaría simplemente con las ganancias patrimoniales de más de un año y el resto, en caso de que existiese, en los cuatro años siguientes.

De acuerdo con lo expuesto se presenta en la figura 11 el diagrama de la operación financiera y en la ecuación 10 se plantea el modelo matemático financiero expresivo de la rentabilidad efectiva $i_{a}$ :

\footnotetext{
29 Su tratamiento fiscal se deriva de lo especificado en los artículos 32, 39-40, 67, 77, 95.1.a y 103.6 del Texto Refundido de la Ley del Impuesto sobre de la Renta de las Personas Físicas, aprobado por el Real Decreto Legislativo 3/2004, del 5 de marzo.

30 Siempre y cuando la diferencia expuesta sea positiva
} 
Yaiza García Padrón

Juan García Boza

Figura 11

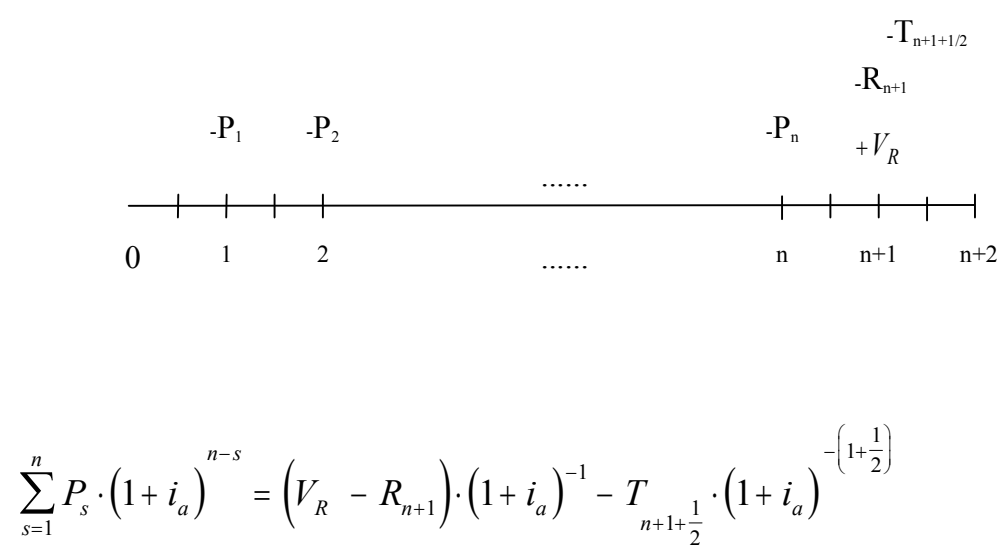

Por último, un caso particular y comparable con los planes de pensiones garantizados es el análisis de la rentabilidad de los fondos de inversión de capitalización garantizados. En este sentido, si no se ejerce la garantía el planteamiento sería idéntico al expuesto; sin embargo, si se ejerce la garantía, la cuota tributaria estaría compuesta por dos partes: una primera parte, derivada de la diferencia entre los valores de reembolso y adquisición, por la que se tributará como variación de patrimonio de acuerdo con lo que se ha analizado; y una segunda parte, devengada por la cuantía que se satisface en concepto de garantía, es decir, por la diferencia entre el valor garantizado y el resultante al final. Por tanto, en este caso el planteamiento sería similar, teniendo presente las particularidades señaladas, al estudiado en los planes de pensiones garantizados.

En síntesis, en el análisis de los fondos de inversión hay que tener presente la modalidad de fondo, reparto o capitalización, dado que su funcionamiento y fiscalidad difieren, mientras que en los primeros se tributa como rendimientos del capital mobiliario, tanto por los dividendos percibidos como por la ganancia derivada de la venta de las participaciones, en los segundos simplemente se tributa por la variación patrimonial que se genera cuando se realiza la citada venta. Además, si se compara la inversión en fondos de inversión con las otras figuras anali- 
Análisis de la rentabilidad financiero-fiscal de los planes de pensiones

y otros productos de captación de ahorro a largo plazo

zadas, se ha de destacar su liquidez y la no existencia de límite para las cuantías invertidas, las cuales no son deducibles y, al igual que los Unit Linked, el riesgo de la inversión lo soporta el inversor, salvo en los fondos garantizados.

Otro punto por resaltar es que, por los rendimientos percibidos de un fondo de inversión de capitalización que se hayan generado en un periodo superior al año se tributa a un tipo fijo del $15 \%$, lo que puede suponer una mayor rentabilidad efectiva para un sujeto, dependiendo de su renta y de la edad a la que realiza la inversión, dado que tanto por los planes de pensiones como por los seguros de vida se tributa al tipo de gravamen del Impuesto sobre la Renta de las Personas Físicas que le corresponda al sujeto, el cual varía en función de la escala en la que se encuentre esa persona.

\section{Conclusiones}

Del análisis financiero-fiscal de los productos financieros y tipos de seguros analizados se deduce que, aunque todos generen el mismo rendimiento, no se puede establecer una única y generalizable jerarquía válida para cualquier sujeto que indique con cual de ellos el ahorrador-inversor obtendrá una mayor rentabilidad efectiva, pues el orden de prelación variará en función de la edad y renta de cada sujeto.

No obstante, se puede afirmar que si el ahorrador-inversor realiza las mismas aportaciones a un plan de pensiones y a un plan de previsión asegurado, la rentabilidad financiero-fiscal del sujeto aparejada a cada operación coincidirá sólo cuando el rendimiento del plan de pensiones sea el mismo que el garantizado por el de previsión.

Por otro lado, señalar que la tributación de los fondos de inversión de capitalización a un tipo fijo del $15 \%$, cuando ha transcurrido más de un año desde que se realizó la inversión, puede conllevar la preferencia por este tipo de fondos para inversiones a medio plazo, frente a los Unit Linked y planes de jubilación donde se tributa al tipo de gravamen que le corresponda al sujeto. 
Yaiza García Padrón

Juan García Boza

Asimismo, el favorecedor tratamiento fiscal de los planes de pensiones en España es en la actualidad el factor que incide en que este producto, en función de las características particulares del sujeto, pueda llevar a una mayor rentabilidad efectiva en comparación con los otros productos analizados.

\section{Bibliografía}

ALVIRA, F., J. García y F. Blanco (1996), "Jubilación, planes de pensiones y opinión pública", en Perspectivas del Sistema Financiero 56, pp. 83-90.

DOMÍNGUEZ, F. y J. López (2004), "Por qué ahorra la gente en planes de pensiones individuales, en Papeles de Trabajo, Serie Economía, Instituto de Estudios Fiscales 27, pp. 1-31.

LEY 40/1998, de 9 de diciembre (BOE de 10 de diciembre), del Impuesto sobre la Renta de las Personas Físicas y otras Normas Tributarias.

LEY 46/2002, de 18 de diciembre (BOE de 19 de diciembre), de reforma parcial del Impuesto sobre la Renta de las Personas Físicas y modifica las Leyes de los Impuestos sobre Sociedades y sobre la Renta de no Residentes.

REAL DECRETO 1775/2004, de 30 de julio (BOE de 4 de agosto), por el que se aprueba el Reglamento del Impuesto sobre la Renta de las Personas Físicas.

REAL DECRETO LEGISLATIVO 1/2002, de 29 de noviembre (BOE de 13 de diciembre), por el que se aprueba el Texto Refundido de la Ley de Regulación de los Planes y Fondos de Pensiones.

REAL DECRETO LEGISLATIVO 3/2004, de 5 de marzo (BOE de 10 de marzo), por el que se aprueba el Texto Refundido de la Ley del Impuesto sobre la Renta de las Personas Físicas.

SÁNCHEZ, F. et al. (1989), Régimen jurídico de los planes y fondos de pensiones, 2a. edición, Española de Seguros, Madrid. 
Análisis de la rentabilidad financiero-fiscal de los planes de pensiones

y otros productos de captación de ahorro a largo plazo

SANZ, E. (1996), "Sistemas de previsión social complementaria", en Perspectivas del Sistema Financiero 56, 53-65.

\section{Anexo: Nomenclatura}

$i_{a}$ : rentabilidad anual financiero-fiscal.

$n$ : número de años durante los cuales el sujeto realiza aportaciones o inversiones (en función del producto financiero de que se trate).

$d$ : número de años de diferimiento en la percepción de las prestaciones.

$h$ : número de años transcurridos desde el origen de la renta constituida por las prestaciones.

$z$ : número de años durante los cuales el sujeto percibe las prestaciones correspondientes.

$t_{s}:$ tipo de gravamen del sujeto en el periodo $s$.

$t_{n+d+h}$ : tipo de gravamen del sujeto en el periodo $n+d+h$.

\section{- Planes de pensiones}

$P_{s}$ : aportación anual al plan de pensiones

$A_{s+\frac{1}{2}}$ : ahorro fiscal derivado de la deducción en el Impuesto sobre la Renta de las aportaciones al plan de pensiones.

$C_{n+d+\dot{h}}$ cuantía bruta de la prestación del plan de pensiones en el periodo $n+d+h$ (cuando la prestación se percibe en forma de renta).

$C_{n+d+h}^{*}$ cuantía neta de la prestación del plan de pensiones cuando se percibe en forma de renta.

$R_{n+d+\dot{r}}$ retención de la prestación bruta del plan de pensiones percibida en forma de renta.

$T_{n+d+h+\frac{1}{2}}$ cuota del Impuesto sobre la Renta derivada del gravamen de la prestación del plan de pensiones percibida en forma de renta.

$C_{n+d+1}^{*}$ :prestación neta del plan de pensiones que se percibe en forma de capital en $n+d+1$.

$C_{n+d+1}$ :prestación bruta del plan de pensiones que se percibe en forma de capital en $n+d+1$.

$T_{n+d++\frac{1}{2}}:$ cuota tributaria derivada del gravamen de la prestación del plan percibida en forma de capital en $n+d+1$. 
Yaiza García Padrón

Juan García Boza

$R_{n+d+1}$ :retención de la prestación bruta del plan de pensiones percibida en forma de capital.

$K^{*}$ : cuantía neta correspondiente a la prestación en forma de capital, percibida del plan de pensiones al principio del año $n+d+1$ (cuando la prestación se percibe en forma mixta).

$T_{n+d+1+\frac{1}{2}}^{\prime}$ :cuota tributaria derivada del gravamen de la prestación del plan de pensiones percibida en forma de capital.

$K_{g}^{*}$ :importe neto de la cuantía diferencia derivada de la garantía que percibe el sujeto al principio del año $n+1$ (en un plan de pensiones garantizado).

$K_{g}$ :importe bruto o íntegro de la cuantía diferencia derivada de la garantía que percibe el sujeto al principio del año $n+1$ (en un plan de pensiones garantizado). $R_{n+1}^{\prime \prime}$ :retención de la cuantía diferencia (en un plan de pensiones garantizado).

$T_{n+1+\frac{i}{2}}{ }^{\prime}$ cuota tributaria derivada del importe percibido por la garantía en el año $n+1$ (en un plan de pensiones garantizado).

\section{- Planes de jubilación}

$P_{s}$ :primas anuales que se satisfacen al plan de jubilación.

$C_{n+d+h}$ :importe bruto de la prestación del plan de jubilación devengada en el año $n+d+h$.

$R_{n}+d+h$ retención derivada de la prestación percibida del plan de jubilación.

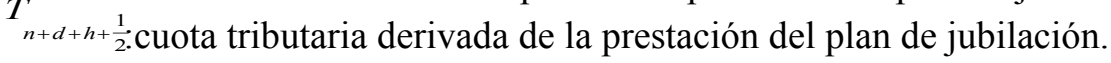

$K$ : cuantía íntegra de la prestación del plan de jubilación que se percibe en forma de capital al principio del año $n+d+1$ (cuando la prestación se percibe en forma mixta).

$R_{n+d+1}^{\prime}$ importe de la retención correspondiente a la prestación del plan de jubilación que se percibe en forma de capital (cuando la prestación se percibe en forma mixta).

$T_{n+d+1+\frac{1}{2}}$ :cuota tributaria correspondiente a la prestación percibida en forma de capital al principio del año $n+d+1$ (cuando la prestación se percibe en forma mixta).

\section{- Unit Linked}

$P_{s}$ : cuantía invertida o prima abonada en el año $s$. 
Análisis de la rentabilidad financiero-fiscal de los planes de pensiones

y otros productos de captación de ahorro a largo plazo

$T:$ : tributación como rendimiento del capital mobiliario por la variación anual del valor liquidativo total de los activos afectos a la póliza.

$C_{n}+1$ : prestación bruta del contrato percibida en $n+1$.

$R_{n}+1$ : retención de la prestación bruta percibida en $n+1$.

$T_{n+d+h+\frac{1}{2}}$ cuota tributaria derivada de la cuantía percibida en $n+1$ (calculada teniendo en cuenta las cuantías por las que se ha tributado a lo largo de la operación).

\section{- Fondos de inversión}

$P_{s}$ : cuantía invertida en el fondo en el año s.

Divs : dividendo bruto anual que reparte el fondo en el año s (en los fondos de inversión de reparto).

$R_{s}$ : retención a cuenta del dividendo percibido en el año s (en los fondos de inversión de reparto).

$T_{s+\frac{1}{2}}$ : cuota tributaria derivada de la cuantía percibida en forma de dividendos en el periodo s (en los fondos de inversión de reparto).

$V_{R}$ : valor de reembolso total percibido por la venta al final del año $n+1$ de las participaciones en el fondo de inversión.

$R_{n+\dot{1}}$ retención por la variación patrimonial derivada de la venta de las participaciones en $n+1$. Esto es, retención del rendimiento generado (de la diferencia entre el precio de venta y el precio de adquisición de las participaciones) con la venta de las participaciones.

$T_{n+1+\frac{1}{2}}$ cuota tributaria derivada de la variación patrimonial generada con la venta de las participaciones. 